and interpreted as the loss of energy by non-elastic percussion of the links of the chain. If we write

$$
\mu=\frac{q}{g} d x
$$

where $\mu$ indicates the mass of the chain element $d x$ and

$$
d Q=\frac{\mu}{2} v^{2}
$$

$\mu$ is also called the reduced mass by percussion of the chain elements (Carnot).

\title{
REFERENCES
}

(1) S. Timoshenko and D. H. Young, Advanced dynamics, McGraw-Hill Book Co., Inc., New York, 1948, pp. 114-116.

(2) P. Frank and R. v. Mises, Die Differential- und Integralgleichungen der Mechanik und Physik, 2. ed., M. S. Rosenberg, New York, 1943, p. 180.

(3) A. G. Greenhill, Proc. Roy. Artillery Inst. 17, 181-226 (1889); or E. Jahnke and F. Emde, Tables of functions, Dover Publications, New York, 1945, pp. 102-104. (The value of $\zeta(u)=3.7174$ for $r=31^{\circ}$ is incorrect. A better value is $\zeta=3.7952$.)

\section{A NOTE ON SUPERSONIC FLOW IN THE TREFFTZ PLANE*}

By JOHN W. MILES (University of California, Los Angeles)

Introduction-The general problem of linearized, supersonic flow has been treated from the standpoint of Fourier transforms in an earlier paper. ${ }^{1}$ The methods set forth therein will be used to calculate the flow conditions far downstream from a lifting surface. If $x$ is the streamwise coordinate, cf. Fig. 1, the downstream plane $x=\infty$ is designated as the Trefftz plane. ${ }^{2}$

This same problem has been treated by Lagerstrom and Graham, ${ }^{3}$ who took advantage of the fact that the flow in the Trefftz plane satisfies Laplace's equation. These same authors also made calculations in regions not infinitely removed from the wing by making use of conical flow methods.

Statement of problem-The vector perturbation velocity due to the presence of the lifting surface is given by

$$
\mathbf{q}(x, y, z)=U \nabla \phi(x, y, z),
$$

where $\phi$ satisfies the linearized equation

$$
\phi_{x x}=\phi_{y y}+\phi_{z z}
$$

${ }^{*}$ Received Feb. 11, 1949.

1J. W. Miles, On linearized supersonic airfoil theory, No. Amer. Avia. Rep. AL-801, Nov., 1948.

${ }^{2}$ E. Trefftz, Zur Prandtlschen Tragflaechentheorie, Math. Ann. 82, 306-319 (1921).

${ }^{3}$ P. A. Lagerstrom and M. E. Graham, Downwash and sidewash induced by three dimensional lifting wings in supersonic flow, Douglas Aircraft Rep. SM-13007, April, 1947. 
the Mach number being taken as $2^{1 / 2}$. The pressure coefficient is given by

$$
\begin{gathered}
c_{p}(x, y)=\frac{p(x, y, 0-)-p(x, y, 0+)}{\rho U^{2} / 2}=4\left(M^{2}-1\right)^{-1 / 2} \gamma(x, y), \\
\gamma(x, y)=\phi_{x}(x, y, 0+) .
\end{gathered}
$$

Orly points in the upper half-space $(z>0)$ are considered, by virtue of the (assumed) asymmetrical nature of the flow. The problem to be solved is the explicit specification

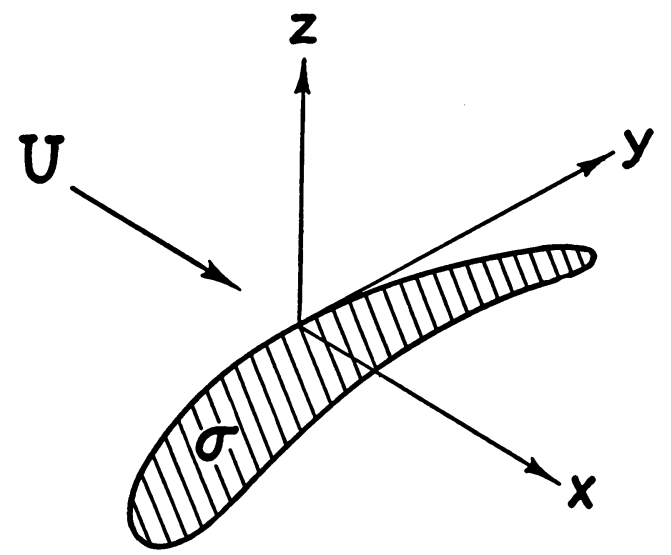

Fig. 1. Coordinate system and lifting surface in the $(x, y)$-plane

of the velocity potential $\phi(\infty, y, z)$ from a knowledge of $\gamma(x, y)$ over a lifting surface $\sigma$ lying in the $(x, y)$-plane ${ }^{4}$ (cf. Fig. 1).

Solution-A solution to Eq. (2), which reduces to $\gamma(x, y)$ at $z=0+$, by virtue of the Fourier transform relations, and vanishes identically upstream, is given by

$$
\begin{gathered}
\phi(x, y, z)=\frac{1}{4 \pi^{2}} \int_{-\infty}^{\infty} d \mu \int_{-\infty}^{\infty} d \nu \int_{-\infty}^{\infty} d \xi \int_{-\infty}^{\infty} d \eta\left[(i \mu)^{-1}+\pi \delta(\mu)\right] \gamma(\xi, \eta) \exp \{i[\mu(x-\xi) \\
\left.\left.+\nu(y-\eta)-\left(\mu^{2}-\nu^{2}\right)^{1 / 2} z\right]\right\},
\end{gathered}
$$

where the path of integration is indented over and under the branch points $+|\mu|$ and $-|\mu|$, respectively, and under the pole $\mu=0$. The presence of the term $\pi \delta(\mu)$ (where $\delta(\mu)$ is the Dirac delta function) in the kernel is required in order that $\phi$ will vanish at $x=-\infty$. The terms $(i \mu)^{-1}$ and $\pi \delta(\mu)$, on the other hand, give equal contributions to the potential at $x=+\infty$, the asymptotic behavior of the solution at $x=\infty$ being determined by the behavior of its Fourier transform at $\mu=0$ (since the integral in the complex $\mu$-plane can be closed in $\mu=+i \infty)$. Thus, it is found that

$$
\phi(\infty, y, z)=\frac{1}{2 \pi} \int_{-\infty}^{\infty} d \nu \int_{-\infty}^{\infty} d \xi \int_{-\infty}^{\infty} d \eta \gamma(\xi, \eta) \exp [i \nu(y-\eta)-|\nu| z] .
$$

\footnotetext{
${ }^{4} \gamma(x, y)$ of course vanishes for $(x, y)$ outside of $\sigma$, since, by hypothesis, only $\sigma$ can support a pressure discontinuity.
} 
While, in many cases, it may be expedient to carry out the integrations with respect to $\xi, \eta$ before integrating with respect to $\nu$, carrying out the latter integration yields ${ }^{5}$

$$
\phi(\infty, y, z)=\frac{z}{\pi} \int_{-\infty}^{\infty} d \xi \int_{-\infty}^{\infty} d \eta \frac{\gamma(\xi, \eta)}{z^{2}+(y-\eta)^{2}} .
$$

This result may also be obtained (or interpreted) through the method of source distributions. In the plane $z=0$, Eq. (7) reduces to

$$
\lim _{z \rightarrow 0+} \phi(\infty, y, z)=\int_{-\infty}^{\infty} d \xi \int_{-\infty}^{\infty} d \eta \gamma(\xi, \eta) \delta(y-\eta)=\int_{-\infty}^{\infty} d \xi \gamma(\xi, y) .
$$

It is of interest to remark that the results, Eqs. (6)-(8), are independent of Mach number and are, therefore, valid for all Mach numbers for which linearized theory is admissible. In view of Jones' results for low aspect ratio airfoils, ${ }^{6}$ this state of affairs is not unexpected.

'B. O. Peirce, A short table of integrals, Ginn and Co., New York, 1929, p. 64.

${ }^{6} \mathrm{R}$. T. Jones, Properties of low-aspect-ratio pointed wings at speeds below and above the speed of sound, NACA TN 1032, March, 1946.

\title{
A THEOREM CONCERNING THE POSITIONS OF EQUILIBRIUM OF A RIGID BODY*
}

\author{
By L. A. MACCOLL (Bell Telephone Laboratories)
}

The following interesting theorem is an immediate consequence of three theorems which are well known in certain mathematical circles. However, since, as far as the writer is aware, the theorem has not previously been stated explicitly, it seems worth while to present it in this note.

Theorem. Let $B$ be a rigid body, of any form, which is free to rotate about a fixed point $P$. Let $B$ be subjected to forces which are derived from a potential energy function $V$, which is an arbitrary single-valued function, of class $C^{2}$, of the coordinates of $B$. Then $B$ has at least four distinct positions of equilibrium.

We first note that the positions (or configurations) of equilibrium of $B$ are the points in the configuration space at which the function $V$, which is defined over the configuration space, is stationary. Then the theorem is an immediate consequence of the following three theorems.

1. The configuration space of a rigid body which is free to rotate about a fixed point is homeomorphic with real three-dimensional projective space.

2. The number of distinct stationary points of a real single-valued function, of class $C^{2}$, defined over a closed manifold $M$ is at least equal to a certain topological invariant of $M$, called the category of $M$.

3 . The category of real $n$-dimensional projective space is $n+1$.

The first of these theorems, in one or another of various equivalent forms, is widely known. In effect, it is given as an exercise on page 56 of Seifert and Threlfall's Lehrbuch der Topologie.

${ }^{*}$ Received Aug. 8, 1949. 